\title{
Tração cutânea intraoperatória para fechamento de ferida após mastectomia bilateral higiênica: relato de caso
}

\author{
Intraoperative skin traction for closure of hygienic bilateral mastectomy wound:
} case report

Jason César Abrantes de

FigueIREDO $^{1}$

Rodrigo Gouvea Rosique ${ }^{2}$

Patricia Jackeline Maciel ${ }^{3}$

Trabalho realizado no Instituto de Cirurgia Plástica Santa Cruz (ICPSC), São Paulo, SP, Brasil.

Artigo submetido pelo SGP (Sistema de Gestão de Publicações) da RBCP.

Artigo recebido: $24 / 7 / 2009$ Artigo aceito: 18/9/2009

\begin{abstract}
RESUMO
Introdução: As sequelas da parede torácica após mastectomia requerem reconstrução por meio de conduta segura e eficiente. Nos casos de recidiva local, sobretudo bilateral, a intervenção cirúrgica deverá ser indicada considerando o estado geral do paciente e a expectativa de vida. A tração intraoperatória é uma técnica de baixa morbidade e rápida execução, reduzindo as cicatrizes decorrentes da utilização de retalhos. Relato do Caso: Paciente do sexo feminino, 45 anos que, após diagnóstico de recidiva tumoral em mama direita e novo tumor em mama esquerda, foi submetida à mastectomia bilateral higiênica com esvaziamento axilar à esquerda. Optou-se pela realização de tração cutânea intraoperatória dos bordos da ferida para fechamento primário da parede torácica devido ao estado geral da paciente. A tração foi realizada conforme a técnica de Góes et al., utilizando-se fios de prolene 2, tracionando a pele por dez minutos, com descanso de dois minutos, até que se obtivesse proximidade suficiente dos bordos das lesões para realização de sutura primária, o que foi alcançado após três ciclos de tração e relaxamento. O procedimento teve duração de duas horas, e foi colocado dreno portovac 3.2, que foi retirado após 7 dias. A paciente evoluiu sem queixas álgicas, sem deiscência ou qualquer outra complicação. Conclusão: A tração intraoperatória demonstrou ser uma técnica segura, funcionalmente eficaz, com menores custo e morbidade, para fechamento de ferida resultante de mastectomia bilateral higiênica.
\end{abstract}

Descritores: Pele. Tração. Mastectomia. Mama/cirurgia.

\section{ABSTRACT}

Introduction: The post-mastectomy chest wall defects require reconstruction by a safe and efficient procedure. In cases of local recurrence, especially bilateral, surgical intervention should be indicated given the patient's general condition and life expectancy. The intraoperative traction is a technique with low morbidity and rapid execution, reducing the scars from the use of flaps. Case Report: Female patient, 45 years old, after diagnosis of recurrence in right breast and a new tumor in left breast, was submitted to hygienic bilateral mastectomy with dissection of left axillary lymph nodes. Opted for intraoperative skin traction of the chest wall wound edges for primary closure due to the general state of the patient. Traction was performed according to the Góes et al. technique, using prolene 2 sutures, stretching the skin for ten minutes, resting for two minutes, until they gain sufficient proximity of the edges of the wound to perform primary suture. This was achieved after three cycles of tension and relaxation. The procedure lasted two hours and was used aspiratory drainage with portovac 3.2 drain, which was removed after 7 days. The patient had no pain complaints, suture without dehiscence or any other complication. Conclusion: The intraoperative traction proved to be a safe, functionally efficient, with lower costs and lower morbidity, to close wounds resulting from hygienic bilateral mastectomy hygienic.

Keywords: Skin. Traction. Mastectomy. Breast/surgery.

1. Doutor em Ciências Médicas pela Faculdade de Medicina da Universidade de São Paulo; Membro Titular da Sociedade Brasileira de Cirurgia Plástica (SBCP); Assistente do Instituto de Cirurgia Plástica Santa Cruz, São Paulo, SP, Brasil.

2. Doutorando em Ciências Médicas pela Faculdade de Medicina de Ribeirão Preto - Universidade de São Paulo; Membro Associado da SBCP; Cirurgião Plástico do Hospital Máster, Goiânia, GO, Brasil.

3. Membro Aspirante em Treinamento da SBCP, Residente do Instituto de Cirurgia Plástica Santa Cruz, São Paulo, SP, Brasil. 


\section{INTRODUÇÃO}

As sequelas da parede torácica após mastectomia requerem reconstrução por meio de conduta segura e eficiente. Nos casos de recidiva local, sobretudo bilateral, a intervenção cirúrgica deverá ser indicada considerando o estado geral do paciente e a expectativa de vida.

A tração intraoperatória é uma técnica de baixa morbidade e rápida execução, reduzindo as cicatrizes decorrentes da utilização de retalhos.

\section{RELATO DO CASO}

Paciente do sexo feminino, 45 anos, com antecedente de câncer gástrico e de colo uterino tratados, foi submetida à mastectomia direita e reconstrução com expansor, em setembro de 2008. Em junho de 2009, após diagnóstico de recidiva tumoral à direita e novo tumor em mama esquerda, foi submetida à mastectomia bilateral higiênica com esvaziamento axilar à esquerda, retirada do expansor e ressecção dos músculos peitorais maior e menor à direita, com margens livres à congelação (Figura 1).

Optou-se pela realização de tração cutânea intraoperatória dos bordos da ferida, para fechamento primário da parede torácica, devido ao estado geral da paciente. A tração foi realizada conforme a técnica de Góes et al. ${ }^{1}$. Penetra-se o fio prolene 2 com agulha de $7,5 \mathrm{~cm}$ em área posterior ao descolamento mínimo, exteriorizando-a no interior da ferida, em direção à margem oposta, onde a aproximadamente 1 $\mathrm{cm}$ da borda transfixa o retalho, sendo então encapado o fio através da agulha com segmento de cateter; retorna-se de maneira inversa, penetrando-se a agulha no final do descolamento e exteriorizando-a o mais distante possível em área não descolada para se completar o "U", bilateralmente. Tracionando-se os dois fios de cada lado, simultaneamente,

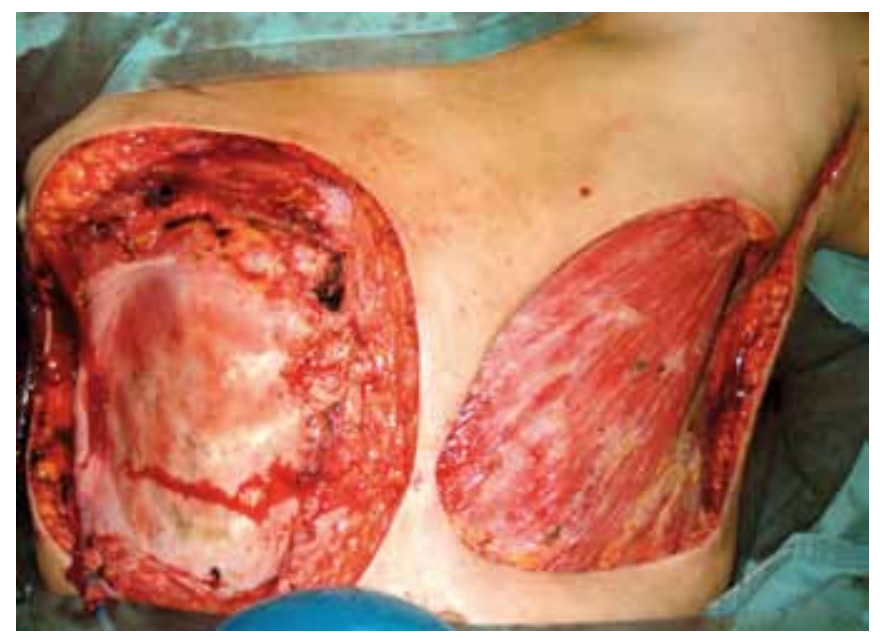

Figura 1 - Ferida após mastectomia higiênica bilateral.

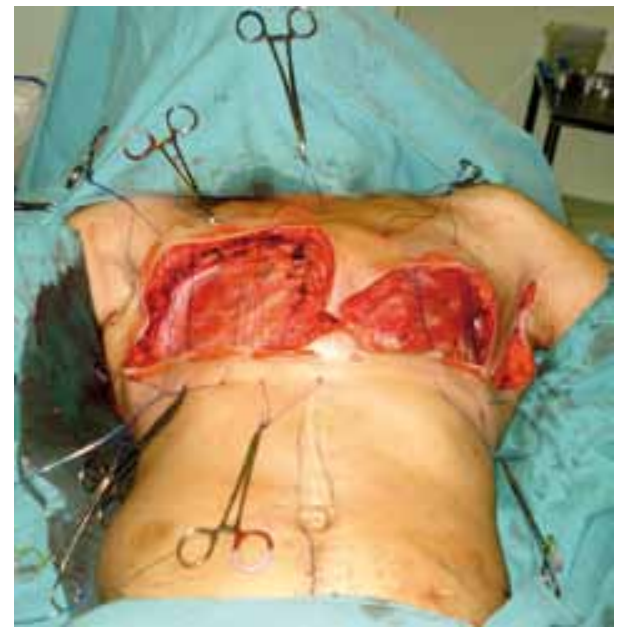

Figura 2 - Relaxamento das bordas por 2 minutos após tração com fios de prolene.

em direções opostas, promove-se uma aproximação das bordas da ferida até um determinado ponto de tensão máxima. Nesse instante, o auxiliar, com quatro pinças Kelly, bloqueia as saídas dos fios rente à pele, para que se mantenha a tensão. Após um período de 10 minutos, soltam-se as pinças Kelly, relaxa-se a tensão por 60 segundos (Figura 2) e realiza-se nova tração. Repete-se este ciclo até que se obtenha proximidade suficiente dos bordos das lesões para realização de sutura primária (Figura 3), sem tensão, com monocryl 4.0 (subdérmico) e mononylon 4.0 (simples separado), o que, no caso relatado, foi alcançado após três ciclos de tração e relaxamento. O procedimento teve duração de duas horas, e foi colocado dreno portovac 3.2, que foi retirado após 7 dias.

A paciente evoluiu sem queixas álgicas, sem deiscência ou qualquer outra complicação (Figura 4).

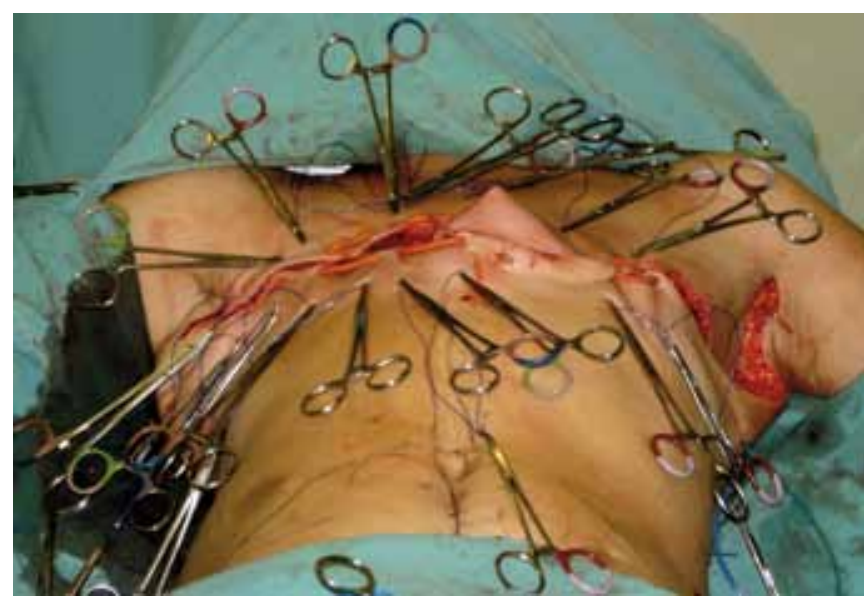

Figura 3 - Tração após 3 ciclos, com afrontamento das bordas da ferida. 


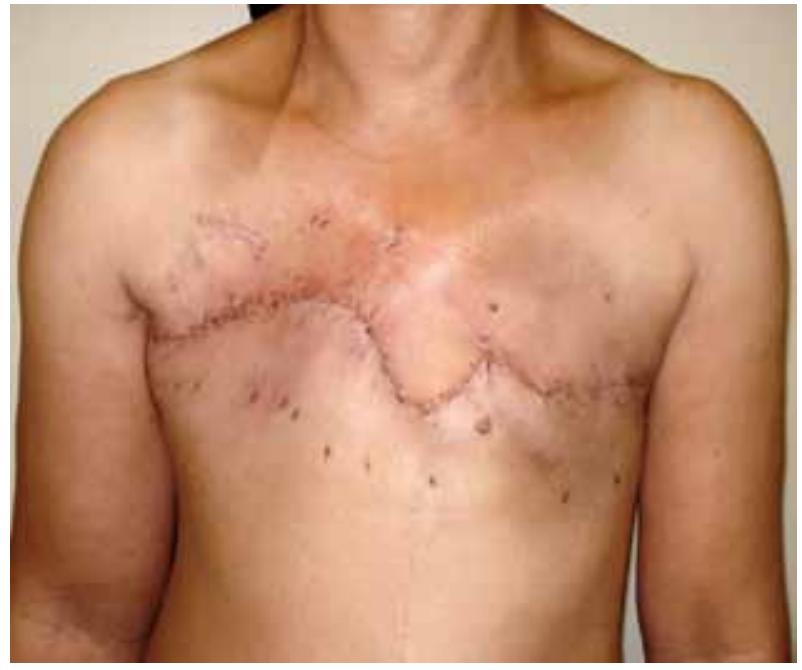

Figura 4 - Cicatriz com 3 semanas de pós-operatório.

\section{DISCUSSÃO}

A tração intraoperatória para estiramento rápido foi criada levando-se em conta as propriedades viscoelásticas da pele ${ }^{2}$, oferecendo uma solução simples para feridas complexas, com menor morbidade em relação a enxertos, expansores e retalhos locais ou livres.

Com esta técnica, a hospitalização é reduzida e os custos do tratamento diminuídos, além de não necessitar de aparelhos de alto custo. Não é recomendado o descolamento grande das bordas da ferida, o que aumentaria o risco de complicações ${ }^{3}$. Não há relato da utilização de tração cutânea para mastectomias bilaterais.

No caso apresentado, a técnica preencheu os requisitos funcionais para mastectomias higiênicas ${ }^{4}$. Houve estabilidade estática e funcional da caixa torácica, sem fenômeno de valvulação ou restrição pulmonar. A técnica ofereceu tecido de cobertura suficientemente denso, com volume e qualidade adequados. Não houve "espaço morto" e coleções, evitandose infecções e restrição torácica por fibrose cicatricial.

\section{CONCLUSÃO}

A tração intraoperatória demonstrou ser uma técnica segura, funcionalmente eficaz, com menores custos e morbidade, para fechamento de ferida resultante de mastectomia bilateral higiênica.

\section{REFERÊNCIAS}

1. Góes CHFS, Kawasaki MC, Mélega JM. Fechamento de feridas por tração cutânea intra-operatória: análise de 23 casos. Rev Soc Bras Cir Plast. 2004;19(2):63-74.

2. Hirshowitz B, Lindenbaum E, Har-Shai Y. A skin-stretching device for the harnessing of the viscoelastic properties of skin. Plast Reconstr Surg. 1993;92(2):260-70.

3. Molea G, Schonauer F, Blasi F. Progressive skin extension: clinical and histological evaluation of a modified procedure using Kirschner wires. Br J Plast Surg. 1999;52(3):205-8.

4. Mendes FH, Freitas AG, Mélega JM. Reconstrução das sequelas de mastectomia e radioterapia. In: Melega JM, Montoro AF, eds. Cirurgia plástica fundamentos e arte: cirurgia reparadora de tronco e membro. Rio de Janeiro:Medsi;2004. p.104-8.

\section{Correspondência para:}

